\title{
Cardiorespiratory Comorbidity and Postoperative Complications following Esophagectomy: a European Multicenter Cohort Study
}

\author{
F. Klevebro, $\mathrm{MD}, \mathrm{PhD}^{1,2}$, J. A. Elliott, $\mathrm{MD}, \mathrm{PhD}^{3,4}$, A. Slaman, $\mathrm{MD}, \mathrm{PhD}^{5}$, B. D. Vermeulen, $\mathrm{MD}, \mathrm{PhD}^{6}$, \\ S. Kamiya, MD, $\mathrm{PhD}^{1,2}$, C. Rosman, MD, $\mathrm{PhD}^{7}$, S. S. Gisbertz, MD, $\mathrm{PhD}^{5}$, P. R. Boshier, MD, $\mathrm{PhD}^{8}$, \\ J. V. Reynolds, MD, $\mathrm{PhD}^{3,4}$, I. Rouvelas, $\mathrm{MD}$, $\mathrm{PhD}^{1,2}$, G. B. Hanna, $\mathrm{MD}, \mathrm{PhD}^{8}$, M. I. van Berge Henegouwen, $\mathrm{MD}$, \\ $\mathrm{PhD}^{5}$, and S. R. Markar, MD, $\mathbf{P h D}^{8}$ \\ ${ }^{1}$ Department of Upper Abdominal Surgery, Centre for Digestive Diseases, Karolinska University Hospital, Stockholm, \\ Sweden; ${ }^{2}$ Division of Surgery, Department of Clinical Science, Intervention and Technology (CLINTEC), Karolinska \\ Institutet, Stockholm, Sweden; ${ }^{3}$ Department of Surgery, Trinity College Dublin, Dublin, Ireland; ${ }^{4}$ The National Esophageal \\ and Gastric Center, St. James's Hospital, Dublin, Ireland; ${ }^{5}$ Department of Surgery, Amsterdam University Medical \\ Centers, Cancer Center Amsterdam, University of Amsterdam, Amsterdam, The Netherlands; ${ }^{6}$ Department of \\ Gastroenterology and Hepatology, Radboud University Medical Center, Nijmegen, The Netherlands; ${ }^{7}$ Radboud University, \\ Nijmegen, The Netherlands; ${ }^{8}$ Department Surgery and Cancer, Imperial College London, London, UK
}

\begin{abstract}
Background. The impact of cardiorespiratory comorbidity on operative outcomes after esophagectomy remains controversial. This study investigated the effect of cardiorespiratory comorbidity on postoperative complications for patients treated for esophageal or gastroesophageal junction cancer.

Patients and Methods. A European multicenter cohort study from five high-volume esophageal cancer centers including patients treated between 2010 and 2017 was conducted. The effect of cardiorespiratory comorbidity and respiratory function upon postoperative outcomes was assessed.

Results. In total 1590 patients from five centers were included; 274 (17.2\%) had respiratory comorbidity, and $468(29.4 \%)$ had cardiac comorbidity. Respiratory comorbidity was associated with increased risk of overall postoperative complications, anastomotic leak, pulmonary complications, pneumonia, increased Clavien-Dindo score, and critical care and hospital length of stay. After neoadjuvant chemoradiotherapy, respiratory comorbidity was associated with increased risk of anastomotic leak [odds
\end{abstract}

(C) The Author(s) 2019

First Received: 10 December 2018;

Published Online: 10 June 2019

F. Klevebro, MD, $\mathrm{PhD}$

e-mail: fredrik.klevebro@ki.se ratio (OR) 1.83, 95\% confidence interval (CI) 1.11-3.04], pneumonia (OR 1.65, 95\% CI 1.10-2.47), and any pulmonary complication (OR 1.52, 95\% CI 1.04-2.22), an effect which was not observed following neoadjuvant chemotherapy or surgery alone. Cardiac comorbidity was associated with increased risk of cardiovascular and pulmonary complications, respiratory failure, and ClavienDindo score $\geq$ IIIa. Among all patients, forced expiratory volume in $1 \mathrm{~s}$ (FEV1)/forced vital capacity (FVC) ratio $>70 \%$ was associated with reduced risk of overall postoperative complications, cardiovascular complications, atrial fibrillation, pulmonary complications, and pneumonia.

Conclusions. The results of this study suggest that cardiorespiratory comorbidity and impaired pulmonary function are associated with increased risk of postoperative complications after esophagectomy performed in highvolume European centers. Given the observed interaction with neoadjuvant approach, these data indicate a potentially modifiable index of perioperative risk.

Surgical resection remains the mainstay of curative treatment for esophageal cancer. ${ }^{1-3}$ Neoadjuvant therapy is increasingly the standard of care for patients with locally advanced disease, with neoadjuvant chemoradiotherapy in the CROSS randomized controlled trial (RCT) achieving 5 -year survival of $47 \%$, establishing a modern benchmark for treatment with curative intent. ${ }^{4}$ Moreover, the recent 
publication of the NeoFLOT data, and presentation of the FLOT 4 trial data, including patients with adenocarcinoma of the esophagogastric junction, highlighted real progress in the curative management of this cancer with pre- and postoperative chemotherapy. ${ }^{5,6}$

Notwithstanding this increased cure rate, esophagectomy remains an exemplar of complex major surgery associated with significant risk of major morbidity, and substantial impact on health-related quality of life. ${ }^{7,8}$ In this context, a number of strategies have been developed in recent years to optimize the perioperative care of patients and to minimize morbidity. These include enhanced recovery after surgery (ERAS) protocols, ${ }^{9,10}$ and centralization of esophageal cancer surgery to high-volume centers. ${ }^{11}$ In addition, the advent of total or hybrid minimally invasive approaches to esophagectomy represents a further advance, with a reduction in postoperative pulmonary infections evidenced in recent randomized trials. $^{12,13}$

An area of controversy is whether the benefit of improved oncologic outcomes from a combination approach including neoadjuvant therapies is offset by an increase in operative complications, in particular pulmonary morbidity. ${ }^{11,14-17}$ In addition, whether preexisting respiratory and cardiac comorbidity impacts on complications for each multimodality treatment approach is unclear. Impaired pulmonary function, measured with spirometry, is a known risk factor for postoperative complications. ${ }^{18}$ Accordingly, the primary objective of this study is to evaluate the effect of cardiac and respiratory comorbidity on postoperative complications and mortality. The secondary objective is to evaluate the role of preoperative pulmonary function tests in identifying patients at risk of postoperative complications and mortality, and to evaluate the effects of cardiorespiratory comorbidity according to treatment approach, either surgery alone, neoadjuvant chemotherapy, or neoadjuvant chemoradiotherapy.

\section{PATIENTS AND METHODS}

\section{Datasets}

Data were collected from 2010 to 2017 from five highvolume European esophageal cancer centers: (1) Karolinska University Hospital, Stockholm, Sweden $(n=400)$, (2) Imperial College London, UK $(n=137)$, (3) Academic Medical Center, Amsterdam, The Netherlands $(n=575)$, (4) Radboud University Medical Center, Nijmegen, The Netherlands $(n=79)$, and (5) The National Esophageal and Gastric Center, St. James's Hospital, Dublin, Ireland $(n=399)$.

\section{Inclusion Criteria}

All patients operated with curative intent for esophageal or esophagogastric junction (EGJ) tumors (Siewert type I and II) were included. Patients receiving surgery alone (S), neoadjuvant chemotherapy (NCS), and neoadjuvant chemoradiotherapy (NCRS) were studied. Patients receiving total minimally invasive, hybrid minimally invasive, and open esophagectomy were included.

\section{Exclusion Criteria}

Patients found to have unresectable disease at time of surgery, those with Siewert type III EGJ tumors, or receiving definitive chemoradiotherapy followed by salvage esophagectomy were excluded.

\section{Clinical Staging and Follow-Up}

Patients with histologically confirmed cancer of the esophagus or EGJ were staged using endoscopy and positron emission tomography (PET)-computed tomography of the thorax and abdomen, which was the standard practice at all centers. Endoscopic ultrasound was used in the staging of EGJ tumors at all centers. All centers had a standardized postoperative pathway following esophagectomy, in keeping with enhanced recovery after surgery (ERAS) principles, though there was some minor heterogeneity between centers.

Follow-up was similar between centers, with mortality identified by linking institutional datasets with national databases to ensure absolute accuracy of all-cause mortality.

\section{Outcomes Including Definition of Complications}

The outcomes evaluated included 30-day and in-hospital complications, which were defined as: postoperative respiratory failure requiring critical care admission, postoperative atrial fibrillation requiring treatment, according to postoperative Clavien-Dindo severity classification, anastomotic leakage or conduit necrosis (endoscopically or radiographically verified), reoperation for any cause, pneumonia defined by the individual investigator when at least one of the following criteria were fulfilled: new and persistent or progressive and persistent radiographic infiltrate, and at least one of: fever $\left(>38.0{ }^{\circ} \mathrm{C}\right.$ or $\left.>100.4^{\circ} \mathrm{F}\right)$, leukopenia $\left(\leq 4000 \mathrm{WBC} / \mathrm{mm}^{3}\right)$, or leukocytosis $\left(>12,000 \mathrm{WBC} / \mathrm{mm}^{3}\right)$, for adults $>70$ years old, altered mental status with no other recognized cause, and at least two of the following: new onset of purulent sputum or change in character of sputum, or increased respiratory secretions, or increased suctioning 
requirements, new-onset or worsening cough, or dyspnea, or tachypnea, rales or bronchial breath sounds, worsening gas exchange [e.g., $\mathrm{O}_{2}$ desaturations (e.g., $\mathrm{PaO}_{2} / \mathrm{FiO}_{2}<$ 240 )], increased oxygen requirements, or increased ventilator demand. ${ }^{19}$ In-hospital, 30-day, and 90-day postoperative all-cause mortality were compiled with complete follow-up. Length of intensive care unit and hospital stay were measured with complete follow-up.

\section{Exposure}

The exposure of the study was cardiac or respiratory disease recorded at baseline. Respiratory disease was defined as chronic pulmonary disease with impaired lung function, including mild, moderate, or severe chronic obstructive lung disease, pulmonary fibrosis, severe asthma, or other chronic pulmonary disease assessed by the surgeon at the initial consultation for esophageal cancer. Cardiac disease was defined as chronic heart disease with impaired cardiac function, including previous myocardial infarction, congestive heart failure, or other chronic cardiac disease assessed by the physician assessing the patient for esophagectomy. Classification of comorbidities was performed by the researcher based on the available clinical data at each site (authors F. Klevebro, J. A. Elliott, A. Slaman, B. D. Vermeulen, P. R. Boshier, and S. R. Markar).

\section{Covariates}

Sex, age, weight, smoking status (smoker/nonsmoker/ ex-smoker), American Society of Anesthesiologists (ASA) grade, Eastern Cooperative Oncology Group (ECOG) performance status, and comorbidities including hypertension and diabetes were collected. Preoperative results on spirometry tests [FVC, FEV1, Tiffeneau-Pinelli index (FEV1/FVC), bicycle or treadmill ergometry results (measured in $\mathrm{W} / \mathrm{kg}$ ), and left ventricular ejection fraction (LVEF) measured by echocardiogram (\%)] were compiled when available. Patients were stratified by FEV1/FVC ratio $>70 \%$ and $\mathrm{LVEF}>55 \% .^{21}$ Operative data including surgical technique were gathered.

\section{Statistical Analysis}

The following confounders with categorizations were used in the adjusted model for the main analysis: age (continuous, years), gender [categorical: male (reference)/ female], histology [categorical: adenocarcinoma (reference)/squamous cell carcinoma], clinical T-stage (categorical: 0-4), clinical N-stage (categorical: 0-3), surgical technique [transthoracic esophagectomy according to Ivor Lewis (reference), three-field esophagectomy according to McKeown, or transhiatal esophagectomy], and surgical approach [open (reference), hybrid minimally invasive, or total minimally invasive esophagectomy].

For categorical outcomes, logistic regression models were used to calculate odds ratios (OR) with $95 \%$ confidence intervals (CI). All above-mentioned regression models were adjusted for predefined confounders.

Approval was granted from the regional research ethics committee of each participating center.

\section{RESULTS}

\section{Comparison of Baseline Patient Demographics} and Pretreatment Tumor Stage

Preoperative Patient Demographics Significant underlying differences in patient age, sex, and ASA score were observed when comparing patients with and without cardiorespiratory comorbidity (Table 1 ).

Pretreatment Tumor Characteristics and Surgical Approach There were no statistically significant differences in tumor stage, tumor type, or use of neoadjuvant treatment between the groups, however proximal tumor location was less common in the cardiorespiratory comorbidity group (Table 1). Surgical technique did show variation between the groups, with the cardiorespiratory comorbidity group having a higher proportion of transhiatal resections $(22.1 \%$ vs. $11.4 \%$, $P<0.001$, Table 1).

\section{Comparison of Postoperative Outcomes}

Respiratory Comorbidity and Postoperative Outcomes Preoperative respiratory comorbidity was associated with an increase in postoperative complications ( $70.8 \%$ vs. $64.4 \%)$, length of intensive care unit stay (median 1 day vs. 0 days), and length of hospital stay (median 18 days vs. 14 days). Postoperative ClavienDindo complication scores were significantly increased among patients with baseline respiratory comorbidity $(P=0.037$, Table 2). Multivariable adjusted analyses showed an increased risk of anastomotic leak (OR 1.64, 95\% CI 1.11-2.41) and pneumonia (OR 1.39, 95\% CI 1.04-1.85, Table 3) among patients with baseline respiratory comorbidity.

Cardiac Comorbidity Patients with preoperative cardiac comorbidity had significantly increased risk of in-hospital mortality ( $5.1 \%$ vs. $2.7 \%$ ), anastomotic leak (15.6 vs. $11.3 \%)$, conduit necrosis ( $4.7 \%$ vs. $0.9 \%)$, cardiovascular complications $(27.6 \%$ vs. $17.7 \%)$, respiratory failure 
TABLE 1 Unadjusted comparison of patient and treatment factors stratified by preoperative cardiorespiratory comorbidity status

\begin{tabular}{|c|c|c|c|c|c|}
\hline$N(\%)$ & $\begin{array}{l}\text { No cardiorespiratory } \\
\text { comorbidity } \\
(N=982)\end{array}$ & $\begin{array}{l}\text { Respiratory } \\
\text { comorbidity } \\
(N=274)\end{array}$ & $\begin{array}{l}\text { Cardiac } \\
\text { comorbidity } \\
(N=468)\end{array}$ & $\begin{array}{l}\text { Cardiorespiratory } \\
\text { comorbidity } \\
(N=608)\end{array}$ & $P$ value* \\
\hline Age (median, IQR), years & $63(56-70)$ & $65(60-71)$ & $67(62-72)$ & $67(61-71)$ & $<0.001$ \\
\hline Sex & & & & & 0.001 \\
\hline Male & $739(75.3)$ & 219 (79.9) & $392(83.8)$ & $500(82.2)$ & \\
\hline Female & $243(24.8)$ & $55(20.1)$ & $76(16.2)$ & $108(17.8)$ & \\
\hline ASA score & & & & & $<0.001$ \\
\hline 1 & $7(0.7)$ & $26(9.5)$ & $24(5.1)$ & $46(7.6)$ & \\
\hline 2 & $345(35.1)$ & $160(58.4)$ & $270(57.7)$ & $356(58.6)$ & \\
\hline 3 & $515(52.4)$ & $87(31.8)$ & $171(36.5)$ & $203(33.4)$ & \\
\hline 4 & $113(11.5)$ & $1(0.4)$ & $3(0.6)$ & $3(0.5)$ & \\
\hline ECOG score $(n=1264)$ & & & & & 0.094 \\
\hline 0 & $582(70.7)$ & $168(74.0)$ & $227(69.4)$ & $319(72.3)$ & \\
\hline 1 & $229(27.8)$ & $51(22.5)$ & $88(26.9)$ & $108(24.5)$ & \\
\hline 2 & $12(1.5)$ & $7(3.1)$ & $11(3.4)$ & $13(3.0)$ & \\
\hline 3 & $0(0)$ & $1(0.4)$ & $1(0.3)$ & $1(0.2)$ & \\
\hline Smoking $(n=850)$ & & & & & 0.086 \\
\hline Never & $158(34.4)$ & 65 (28.4) & $76(27.2)$ & $107(27.4)$ & \\
\hline Former & $211(46.0)$ & $104(45.4)$ & $157(56.3)$ & $200(51.2)$ & \\
\hline Currently & $90(19.6)$ & $60(26.2)$ & $46(16.5)$ & $84(21.5)$ & \\
\hline FVC L, median (IQR) & $4.1(3.2-4.7)$ & $3.7(3.1-4.4)$ & $3.8(3.4-4.3)$ & $3.7(3.2-4.3)$ & 0.008 \\
\hline FEV1 L, median (IQR) & $2.9(2.4-3.5)$ & $2.4(1.9-3.1)$ & $2.8(2.1-3.1)$ & $2.6(2.1-3.1)$ & 0.020 \\
\hline $\begin{array}{l}\text { FEV1/FVC median \% (IQR) } \\
(n=850)\end{array}$ & $83.3(74.3-97.0)$ & $73.3(63.2-82.6)$ & $88.0(75.9-100)$ & $84(71.7-98.0)$ & 0.747 \\
\hline Bicycle test $(\mathrm{W})(n=34)$ & No observations & $129(114-156)$ & $131(118-157)$ & $131(117-160)$ & \\
\hline $\begin{array}{l}\text { Left ventricular ejection } \\
\text { fraction }(\%)(n=234)\end{array}$ & $59.5(55.0-63.0)$ & $59(55.0-60.0)$ & $55(50.0-60.0)$ & $56.5(53.0-60.0)$ & 0.056 \\
\hline $\mathrm{cT}$ & & & & & 0.127 \\
\hline 1 & $111(11.3)$ & $44(16.1)$ & $62(13.3)$ & $81(13.3)$ & \\
\hline 2 & 173 (17.6) & $42(15.3)$ & $75(16.0)$ & $103(16.9)$ & \\
\hline 3 & $614(62.5)$ & $168(61.3)$ & $308(65.8)$ & $392(64.5)$ & \\
\hline 4 & $48(4.9)$ & $12(4.4)$ & $10(2.1)$ & $18(3.0)$ & \\
\hline$X$ & $36(3.7)$ & $8(2.9)$ & $13(2.8)$ & $14(2.3)$ & \\
\hline $\mathrm{cN}$ & & & & & 0.057 \\
\hline 0 & $438(44.6)$ & $125(45.6)$ & $182(38.9)$ & $244(40.1)$ & \\
\hline 1 & $394(40.1)$ & $121(44.2)$ & $224(47.9)$ & $286(47.0)$ & \\
\hline 2 & $133(13.5)$ & $22(8.0)$ & $57(12.2)$ & $69(11.4)$ & \\
\hline 3 & $17(1.7)$ & $6(2.2)$ & $5(1.1)$ & $9(1.5)$ & \\
\hline Tumor type & & & & & 0.125 \\
\hline Adenocarcinoma & $747(76.1)$ & $209(76.3)$ & $367(78.2)$ & $475(78.1)$ & \\
\hline $\mathrm{SCC}$ & $207(21.1)$ & $62(22.6)$ & $94(20.1)$ & $125(20.6)$ & \\
\hline Other & $28(2.9)$ & $3(1.1)$ & $7(1.5)$ & $8(1.3)$ & \\
\hline Tumor location & & & & & $<0.001$ \\
\hline Upper/middle & $142(14.5)$ & $35(12.8)$ & $48(10.3)$ & $66(10.9)$ & \\
\hline Distal & $326(33.2)$ & $88(32.1)$ & $253(54.1)$ & $297(48.9)$ & \\
\hline EGJ & $514(52.3)$ & $151(55.1)$ & $167(35.7)$ & $245(40.3)$ & \\
\hline Surgical approach & & & & & 0.005 \\
\hline
\end{tabular}


TABLE 1 continued

\begin{tabular}{lllll}
\hline$N(\%)$ & $\begin{array}{l}\text { No cardiorespiratory } \\
\text { comorbidity } \\
(N=982)\end{array}$ & $\begin{array}{l}\text { Respiratory } \\
\text { comorbidity } \\
(N=274)\end{array}$ & $\begin{array}{l}\text { Cardiac } \\
\text { comorbidity } \\
(N=468)\end{array}$ & $\begin{array}{l}\text { Cardiorespiratory } \\
\text { comorbidity } \\
(N=608)\end{array}$ \\
\hline Open & $527(53.7)$ & $194(70.8)$ & $223(47.7)$ & $338(55.6)$ \\
HMIO & $36(3.7)$ & $5(1.8)$ & $5(1.1)$ & $6(1.0)$ \\
TMIO & $419(42.7)$ & $75(27.4)$ & $240(51.3)$ & $264(43.4)$ \\
Surgical technique & $564(57.5)$ & $139(51.1)$ & $228(48.8)$ & $293(48.4)$ \\
Ivor Lewis & $305(31.1)$ & $62(22.8)$ & $140(30.0)$ & $179(29.5)$ \\
McKeown & $112(11.4)$ & $71(26.1)$ & $99(21.2)$ & $134(22.1)$ \\
Transhiatal & & $81(29.6)$ & $105(22.4)$ & $137(22.5)$ \\
Neoadjuvant treatment & $232(23.6)$ & $54(19.7)$ & $54(11.5)$ & $90(14.8)$ \\
None & $182(18.5)$ & $139(50.7)$ & $309(66.0)$ & $381(62.7)$ \\
Chemotherapy & $568(57.8)$ & & & 0.001 \\
Chemoradiotherapy & & & & \\
\hline
\end{tabular}

$I Q R$ interquartile range, $H M I O$ hybrid minimally invasive esophagectomy, $T M I O$ totally minimally invasive esophagectomy, SCC squamous cell carcinoma

*Comparing patients with and without preoperative cardiopulmonary disease

(14.1\% vs. $9.7 \%)$, reoperation for any cause $(11.8 \%$ vs. $7.1 \%)$, and any pulmonary complication $(42.7 \%$ vs. $34.9 \%)$. Postoperative Clavien-Dindo complication scores were also increased with cardiac comorbidity $(P<0.001$, Table 2). Multivariable adjusted analyses showed an increased risk of cardiovascular complications (OR 1.63, 95\% CI 1.25-2.13), pulmonary complications (OR 1.44, 95\% CI 1.14-1.82), respiratory failure (OR 1.49, 95\% CI 1.06-2.09), and Clavien-Dindo score $\geq$ IIIa (OR 1.73, 95\% CI 1.34-2.25, Table 3) among patients with preoperative cardiac comorbidity.

\section{Cardiorespiratory Comorbidity among Postoperative} Complications According to Neoadjuvant Protocol In a multivariable adjusted analysis, respiratory comorbidity was associated with increased risk of anastomotic leak (OR 1.83, 95\% CI 1.11-3.04), pneumonia (OR 1.65, 95\% CI 1.10-2.47), and any pulmonary complication (OR 1.52, 95\% CI 1.04-2.22) after neoadjuvant chemoradiotherapy but not after neoadjuvant chemotherapy or surgery alone (Table 4).

Cardiac comorbidity was associated with increased risk of cardiovascular complications, pulmonary complications, and Clavien-Dindo score $\geq$ IIIa regardless of neoadjuvant treatment. In the neoadjuvant chemotherapy group, cardiac comorbidity was associated with increased risk of in-hospital mortality (OR 12.62, 95\% CI 1.30-117.80, Table 4).

\section{Preoperative Respiratory and Cardiac Investigations and Postoperative Outcomes}

Of 1590 patients, $608(38.2 \%)$ had a cardiorespiratory comorbidity, of whom $407(66.9 \%)$ had documented preoperative pulmonary function testing and $106(17.4 \%)$ had a preoperative echocardiogram. Ergometry results were only available for $34(5.6 \%)$ patients and were not included in the analyses. Unadjusted analyses showed that FEV1/FVC ratio $>70 \%$ was associated with reduced overall postoperative complications, cardiovascular complications, atrial fibrillation, pulmonary complications, and pneumonia. Adjusted analyses showed that FEV1/FVC ratio $>70 \%$ was associated with reduced risk of overall postoperative complications (OR $0.57,95 \%$ CI 0.37-0.89) and atrial fibrillation (OR 0.46 , 95\% CI $0.28-0.75$, Table 5). LVEF $>55 \%$ was associated with decreased risk of anastomotic leak (adjusted OR 0.34, 95\% CI 0.12-0.95), but was otherwise unrelated to postoperative outcomes.

\section{DISCUSSION}

This large multicenter cohort study from high-volume European academic centers highlights several important points. First, cardiac or respiratory comorbidity substantially increased the risk and severity of postoperative complications, among patients deemed fit ab initio for surgery. Second, impaired pulmonary function based on spirometry reliably predicted pulmonary and cardiovascular complications, atrial fibrillation, pneumonia, and overall postoperative complications. Third, neoadjuvant chemoradiotherapy was associated with increased risk of anastomotic leak and pulmonary complications among patients with respiratory comorbidity, while neoadjuvant chemotherapy or surgery alone was not. 
TABLE 2 Comparison of postoperative outcomes stratified by preoperative cardiorespiratory comorbidity status

\begin{tabular}{|c|c|c|c|c|c|}
\hline$N(\%)$ & $\begin{array}{l}\text { No cardiorespiratory } \\
\text { comorbidity } \\
(N=982)\end{array}$ & $\begin{array}{l}\text { Respiratory } \\
\text { comorbidity } \\
(N=274)\end{array}$ & $\begin{array}{l}\text { Cardiac } \\
\text { comorbidity } \\
(N=468)\end{array}$ & $\begin{array}{l}\text { Cardiorespiratory } \\
\text { comorbidity } \\
(N=608)\end{array}$ & $P$ value* \\
\hline In-hospital mortality & $28(2.9)$ & $12(4.4)$ & $24(5.1)^{* *}$ & $26(4.3)$ & 0.128 \\
\hline Postoperative complication & $632(64.4)$ & $194(70.8)^{* *}$ & $305(65.2)$ & $408(67.1)$ & 0.263 \\
\hline $\begin{array}{l}\text { Length of intensive care unit } \\
\text { stay, median days (IQR) }\end{array}$ & $0(0-2)$ & $1(0-5)^{* *}$ & $1(0-5)$ & $1(0-5)^{* *}$ & $<0.001$ \\
\hline $\begin{array}{l}\text { Length of hospital stay, median } \\
\text { days (IQR) }\end{array}$ & $14(10-22)$ & $18(12-30)^{* *}$ & $15(9-27)$ & $15(10-27)$ & 0.057 \\
\hline Reoperation & $48(7.1)$ & $20(10.7)$ & $47(11.8)^{* *}$ & $59(11.4)^{* *}$ & 0.010 \\
\hline Pneumonia & $255(26.0)$ & $98(35.8)^{* *}$ & $136(29.1)$ & $180(29.6)$ & 0.114 \\
\hline Anastomotic leak & $111(11.3)$ & $42(15.3)$ & $73(15.6)^{* *}$ & $86(14.1)$ & 0.095 \\
\hline Conduit necrosis & $6(0.9)$ & $2(2.7)$ & $19(4.7)^{* *}$ & $19(3.7)^{* *}$ & 0.001 \\
\hline Cardiovascular complication & $174(17.7)$ & $57(20.9)$ & $129(27.6)^{* *}$ & $153(25.2)^{* *}$ & $<0.001$ \\
\hline Atrial fibrillation & $109(11.1)$ & $32(11.7)$ & $49(10.5)$ & $67(11.0)$ & 0.961 \\
\hline Respiratory failure & $95(9.7)$ & $41(15.0)^{* *}$ & $66(14.1)^{* *}$ & $81(13.3)^{* *}$ & 0.024 \\
\hline Pulmonary complication & $343(34.9)$ & $122(44.5)^{* *}$ & $200(42.7)^{* *}$ & $254(41.8)^{* *}$ & 0.006 \\
\hline Clavien-Dindo score & - & $P=0.037 * *$ & $P<0.001 * *$ & & $P<0.001$ \\
\hline Grade I & $216(28.0)$ & $64(26.7)$ & $75(26.9)$ & $105(22.8)$ & \\
\hline Grade II & $231(29.9)$ & $68(28.3)$ & $76(22.2)$ & $114(24.7)$ & \\
\hline Grade IIIa & $143(18.5)$ & $27(11.3)$ & $46(13.5)$ & $64(13.9)$ & \\
\hline Grade IIIb & $56(7.3)$ & $22(9.2)$ & $19(5.6)$ & $30(6.5)$ & \\
\hline Grade IVa & $93(12.1)$ & $45(18.8)$ & $84(24.6)$ & $100(21.7)$ & \\
\hline Grade IVb & $25(3.2)$ & $13(5.4)$ & $26(7.6)$ & $32(6.9)$ & \\
\hline Grade V & $8(1.0)$ & $1(0.42)$ & $16(4.7)$ & $16(3.5)$ & \\
\hline $\begin{array}{l}\text { Clavien-Dindo score, median } \\
\text { (IQR) }\end{array}$ & II (I-IIIa) & II (I-IIIb) & IIIa (II-IVa) & IIIa (II-IVa) & $<0.001$ \\
\hline
\end{tabular}

*Comparing patients with and without preoperative cardiopulmonary disease

$* * P<0.05$ comparing patients with and without preoperative cardiopulmonary disease

Although risk assessment as part of modern management and ERAS programs for esophageal surgery has become increasingly standardized, accurate prediction of risk remains a significant clinical challenge. ${ }^{11,17}$ Age alone has not been associated with increased postoperative risk in most studies. ${ }^{22,23}$ Higher ASA score and decreased performance status are however established risk factors, and this was confirmed in the present study (data not shown). ${ }^{11}$ Preoperative assessment using spirometry, or bicycle or treadmill ergometry is commonly used to evaluate the patient's fitness to undergo esophagectomy. ${ }^{18,24}$ Interestingly, however, even among academic centers, approximately only two-thirds of patients with preoperative cardiac or respiratory disease underwent such evaluation in this time period. For those measures, FEV1/ FVC ratio $>70 \%$ was independently associated with a decreased risk of overall postoperative complications, cardiovascular complications, atrial fibrillation, pulmonary complications, and pneumonia. It seems prudent that all patients undergoing treatment with curative intent for esophageal cancer should undergo pulmonary physiology studies, ideally before and after neoadjuvant therapy. Cardiopulmonary exercise testing (CPET) applies analysis of breath-by-breath expired gas data to evaluate cardiorespiratory capacity. This test might provide a more accurate and useful estimate of cardiorespiratory function and risks associated with esophageal cancer treatment. ${ }^{25,26}$ The 6-min walk test is another test of cardiorespiratory capacity and physical function, which have been shown to predict perioperative risk in thoracic surgery. ${ }^{27,28}$ The role of these tests in esophageal cancer treatment should be evaluated in prospective studies and in trials of prehabilitation.

Moreover, based on the present data, studies targeting preoperative optimization through prehabilitation programs, including exercise interventions and smoking cessation, to improve pulmonary function, have considerable therapeutic rationale. A randomized trial that assessed 

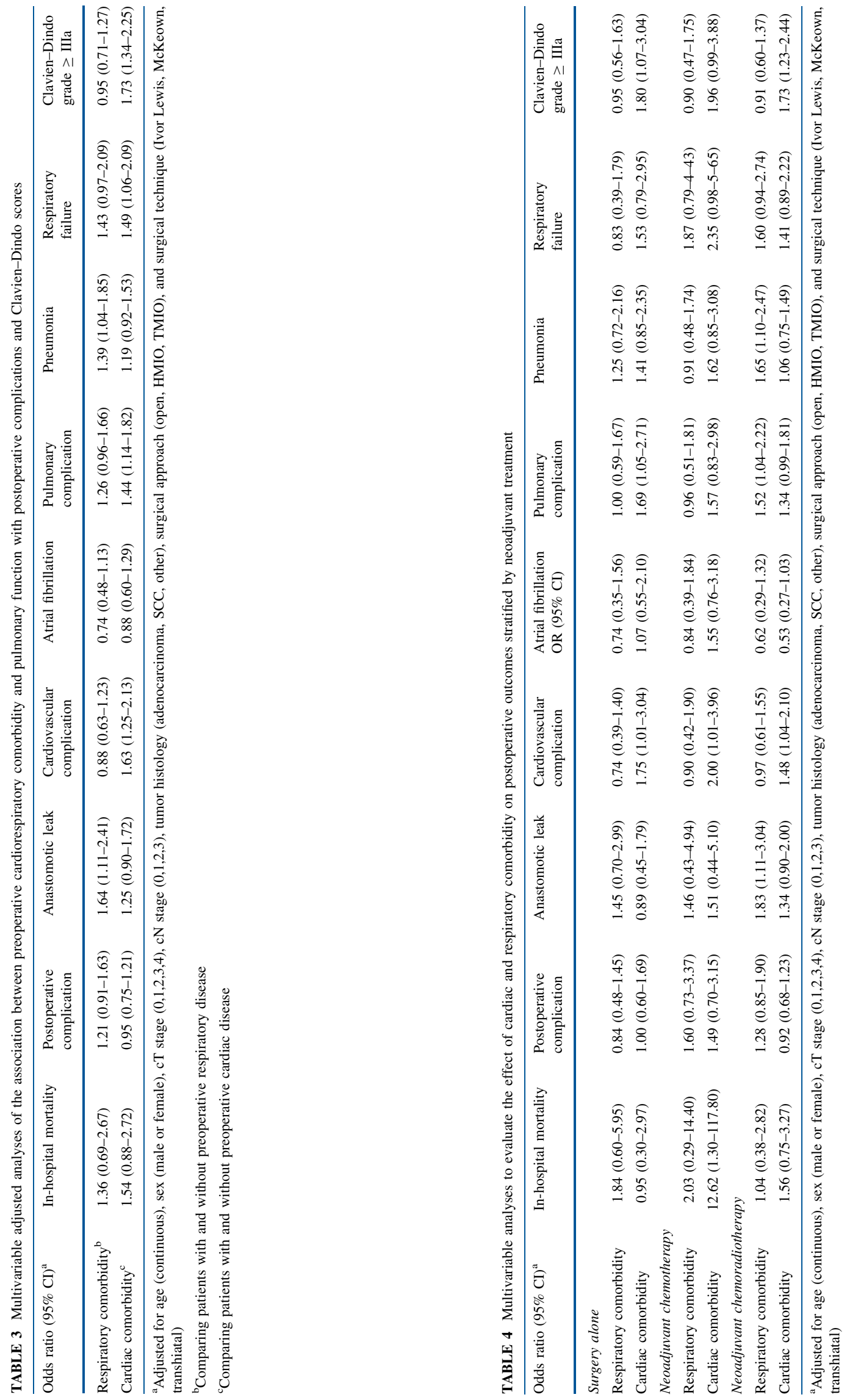
the effect of preoperative inspiratory muscle training showed improved inspiratory muscle strength but no difference in lung function parameters or pulmonary complications. $^{29}$ A recent randomized controlled trial showed that prehabilitation with an exercise program and nutritional support significantly increased patients' results on the 6-min walk test. ${ }^{30}$ Preoperative optimization with exercise, nutritional support, and the interaction with cardiorespiratory comorbidity represent a highly pertinent future research area.

In this study, cardiac comorbidity was associated with increased in-hospital mortality after neoadjuvant chemotherapy and esophagectomy. New-onset atrial fibrillation may be the most common specific complication after esophagectomy, affecting approximately $20 \%$ of patients. $^{31}$ Preoperative treatment with 5-fluorouracil (5FU) and platins is known to cause cardiac sensitization. ${ }^{32}$ It is interesting that this was not seen in the NCRS group, a fact that might be explained by the differences in the chemotherapy regimens which are used concomitant with radiotherapy. Furthermore, cytotoxic agents may impair myocellular proliferation by disrupting the mammalian target of rapamycin kinase pathway, ${ }^{33}$ while cisplatin also negatively impacts muscle function through a number of mechanisms including impaired Akt phosphorylation, leading to sustained activation of the degradative proteasome and autophagy systems, and altered nuclear factor kappa-light-chain-enhancer of activated B cells (NF-kB) signaling. ${ }^{34,35}$ Together these mechanisms may contribute to myocardial dysfunction among patients treated with preoperative chemotherapy. ${ }^{24,32}$ There was an increased risk for anastomotic leak, pulmonary complications, and pneumonia for patients with respiratory comorbidity after NCRS but not after NCS. This could be an effect of the added radiotherapy, which has been shown in previous studies to be associated with increased risk for radiation pneumonitis, reduced physical endurance, pulmonary diffusion capacity, and heart function, and increased risk for severe postoperative complications after esophagectomy compared with NCS. ${ }^{14,17,36-42}$ Modern neoadjuvant chemoradiotherapy regimens for esophageal cancer appear to result in specific reductions in pulmonary diffusion capacity, particularly among older adults and those with a history of smoking or preexisting lung disease, which may limit such patients' ability to tolerate pulmonary morbidity, increasing the risk of postoperative respiratory failure. ${ }^{42}$ Detailed analysis of postoperative outcomes is embedded in ongoing randomized controlled trials comparing NCRS with NCS for patients with esophageal adenocarcinoma, ${ }^{43,44}$ and these secondary outcome measures will be of critical importance, particularly if there is oncologic equivalence. 
The limitations of this study include the retrospective design and the difficulties in defining and measuring postoperative outcomes, however it must be noted that coding of complications was standardized for this study in accordance with international consensus recommendations. ${ }^{45}$ The included patients have been evaluated according to predefined variables by a devoted researcher at each participating center to increase the internal validity of the study. Pretreatment cardiac function tests were not available in the majority of patients, and cardiorespiratory comorbidity was assessed clinically. It is possible that cardiorespiratory comorbidity status may have been misclassified in some patients. Pulmonary function tests (PFTs) were however available in $67 \%$ of the patients. This could lead to understatement of the positive associations in the study, and prevents stratified analyses by type and function of the cardiorespiratory comorbidities. The small number of patients receiving preoperative cardiac investigations prevented robust investigation of the link between objective measurements of cardiac function and postoperative outcome. Furthermore, diffusion capacity for carbon monoxide (DLCO) was often not available, which prevented meaningful analyses of the association with postoperative complications which has been demonstrated in previous research. ${ }^{46}$ Strengths of the study include the standardized classification of complications, the large sample size, and the multicenter design conferring external validity to the study within high-volume European centers.

In conclusion, this study shows that cardiorespiratory comorbidity is a clear risk factor for postoperative complications after esophagectomy. Impaired preoperative lung function is associated with increased risk of postoperative complications, particularly following neoadjuvant chemoradiotherapy. Careful clinical assessment, with thorough investigation of cardiorespiratory function, is required to facilitate treatment planning for patients considered for multimodal therapy for locally advanced esophageal cancer.

OPEN ACCESS This article is distributed under the terms of the Creative Commons Attribution 4.0 International License (http://crea tivecommons.org/licenses/by/4.0/), which permits unrestricted use, distribution, and reproduction in any medium, provided you give appropriate credit to the original author(s) and the source, provide a link to the Creative Commons license, and indicate if changes were made.

\section{REFERENCES}

1. Pennathur A, Gibson MK, Jobe BA, et al. Oesophageal carcinoma. Lancet. 2013; 381(9864):400-12.

2. Sundelof M, Ye W, Dickman PW, et al. Improved survival in both histologic types of oesophageal cancer in Sweden. Int J Cancer. 2002; 99(5):751-4.
3. Rouvelas I, Zeng W, Lindblad M, et al. Survival after surgery for oesophageal cancer: a population-based study. Lancet Oncol. 2005; 6(11):864-70.

4. van Hagen P, Hulshof MC, van Lanschot JJ, et al. Preoperative chemoradiotherapy for esophageal or junctional cancer. $N$ Engl $J$ Med. 2012; 366(22):2074-84.

5. Al-Batran SE, Hofheinz RD, Pauligk C, et al. Histopathological regression after neoadjuvant docetaxel, oxaliplatin, fluorouracil, and leucovorin versus epirubicin, cisplatin, and fluorouracil or capecitabine in patients with resectable gastric or gastro-oesophageal junction adenocarcinoma (FLOT4-AIO): results from the phase 2 part of a multicentre, open-label, randomised phase 2/3 trial. Lancet Oncol. 2016; 17(12):1697-1708.

6. Al-Batran SE, Homann N, Pauligk C, et al. Perioperative chemotherapy with fluorouracil plus leucovorin, oxaliplatin, and docetaxel versus fluorouracil or capecitabine plus cisplatin and epirubicin for locally advanced, resectable gastric or gastro-oesophageal junction adenocarcinoma (FLOT4): a randomised, phase 2/3 trial. Lancet 2019; 393(10184):1948-1957.

7. Schieman C, Wigle DA, Deschamps C, et al. Patterns of operative mortality following esophagectomy. Dis Esophagus. 2012; 25(7):645-51.

8. Paul S, Altorki N. Outcomes in the management of esophageal cancer. J Surg Oncol. 2014; 110(5):599-610.

9. Wang L, Zhu C, Ma X, et al. Impact of enhanced recovery program on patients with esophageal cancer in comparison with traditional care. Support Care Cancer. 2017; 25(2):381-9.

10. Gemmill EH, Humes DJ, Catton JA. Systematic review of enhanced recovery after gastro-oesophageal cancer surgery. Ann $R$ Coll Surg Engl. 2015; 97(3):173-9.

11. Markar S, Gronnier C, Duhamel A, et al. Pattern of postoperative mortality after esophageal cancer resection according to center volume: results from a large European multicenter study. Ann Surg Oncol. 2015; 22(8):2615-23.

12. Biere SS, van Berge Henegouwen MI, Maas KW, et al. Minimally invasive versus open oesophagectomy for patients with oesophageal cancer: a multicentre, open-label, randomised controlled trial. Lancet. 2012; 379(9829):1887-92.

13. Mariette C, Markar SR, Dabakuyo-Yonli TS, et al. Hybrid minimally invasive esophagectomy for esophageal cancer. $N$ Engl J Med. 2019; 380(2):152-62.

14. Avendano CE, Flume PA, Silvestri GA, et al. Pulmonary complications after esophagectomy. Ann Thorac Surg. 2002; 73(3):922-6.

15. Klevebro F, Johnsen G, Johnson E, et al. Morbidity and mortality after surgery for cancer of the oesophagus and gastro-oesophageal junction: a randomized clinical trial of neoadjuvant chemotherapy vs. neoadjuvant chemoradiation. Eur J Surg Oncol. 2015; 41(7):920-6.

16. Stahl M, Walz MK, Stuschke M, et al. Phase III comparison of preoperative chemotherapy compared with chemoradiotherapy in patients with locally advanced adenocarcinoma of the esophagogastric junction. J Clin Oncol. 2009; 27(6):851-6.

17. Kumagai K, Rouvelas I, Tsai JA, et al. Meta-analysis of postoperative morbidity and perioperative mortality in patients receiving neoadjuvant chemotherapy or chemoradiotherapy for resectable oesophageal and gastro-oesophageal junctional cancers. Br J Surg. 2014; 101(4):321-38.

18. Ferguson MK, Durkin AE. Preoperative prediction of the risk of pulmonary complications after esophagectomy for cancer. $J$ Thorac Cardiovasc Surg. 2002; 123(4):661-9.

19. Low DE, Kuppusamy MK, Alderson D, et al. Benchmarking complications associated with esophagectomy. Ann Surg. 2019; 269(2):291-298. 
20. Mohamed Hoesein FA, Zanen P, Lammers JW. Lower limit of normal or FEV1/FVC $<0.70$ in diagnosing COPD: an evidencebased review. Respir Med. 2011; 105(6):907-15.

21. Fonarow GC, Hsu JJ. Left ventricular ejection fraction: what is "normal"? JACC Heart Fail. 2016; 4(6):511-3.

22. Ferguson MK, Martin TR, Reeder LB, et al. Mortality after esophagectomy: risk factor analysis. World J Surg. 1997; 21(6):599-603; discussion 603-4.

23. Law S, Wong KH, Kwok KF, et al. Predictive factors for postoperative pulmonary complications and mortality after esophagectomy for cancer. Ann Surg. 2004; 240(5):791-800.

24. von Dobeln GA, Nilsson M, Adell G, et al. Pulmonary function and cardiac stress test after multimodality treatment of esophageal cancer. Pract Radiat Oncol. 2016; 6(3):e53-9.

25. Levett DZH, Jack S, Swart M, et al. Perioperative cardiopulmonary exercise testing (CPET): consensus clinical guidelines on indications, organization, conduct, and physiological interpretation. Br J Anaesth. 2018; 120(3):484-500.

26. Richardson K, Levett DZH, Jack S, et al. Fit for surgery? Perspectives on preoperative exercise testing and training. $\mathrm{Br} J$ Anaesth. 2017; 119(suppl_1):i34-43.

27. Enright PL. The six-minute walk test. Respir Care. 2003; 48(8):783-5.

28. Holden DA, Rice TW, Stelmach K, et al. Exercise testing, 6-min walk, and stair climb in the evaluation of patients at high risk for pulmonary resection. Chest. 1992; 102(6):1774-9.

29. Valkenet K, Trappenburg JCA, Ruurda JP, et al. Multicentre randomized clinical trial of inspiratory muscle training versus usual care before surgery for oesophageal cancer. Br J Surg. 2018; 105(5):502-11.

30. Minnella EM, Awasthi R, Loiselle SE, et al. Effect of exercise and nutrition prehabilitation on functional capacity in esophagogastric cancer surgery: a randomized clinical trial. JAMA Surg. 2018; 153(12):1081-1089. https://doi.org/10.1001/jamasurg.201 8.1645 .

31. Mc Cormack O, Zaborowski A, King S, et al. New-onset atrial fibrillation post-surgery for esophageal and junctional cancer: incidence, management, and impact on short- and long-term outcomes. Ann Surg. 2014; 260(5):772-8; discussion 778.

32. Tsibiribi P, Descotes J, Lombard-Bohas C, et al. Cardiotoxicity of 5-fluorouracil in 1350 patients with no prior history of heart disease. Bull Cancer. 2006; 93(3):E27-30.

33. Fearon K, Arends J, Baracos V. Understanding the mechanisms and treatment options in cancer cachexia. Nat Rev Clin Oncol. 2013; 10(2):90-9.

34. Fanzani A, Zanola A, Rovetta F, et al. Cisplatin triggers atrophy of skeletal $\mathrm{C} 2 \mathrm{C} 12$ myotubes via impairment of Akt signalling pathway and subsequent increment activity of proteasome and autophagy systems. Toxicol Appl Pharmacol. 2011; 250(3):312-21.

35. Damrauer SM, Acharyya S, Baldwin AS, Couch ME, Guttridge DC Chemotherapy-induced muscle wasting: association with NF$\kappa \mathrm{B}$ and cancer cachexia. Basic Appl Myology. 2008; 18(5):139-48.
36. Lund M, Alexandersson von Dobeln G, Nilsson M, et al. Effects on heart function of neoadjuvant chemotherapy and chemoradiotherapy in patients with cancer in the esophagus or gastroesophageal junction-a prospective cohort pilot study within a randomized clinical trial. Radiat Oncol. 2015; 10(1):16.

37. Liedman B, Johnsson E, Merke C, et al. Preoperative adjuvant radiochemotherapy may increase the risk in patients undergoing thoracoabdominal esophageal resections. Dig Surg. 2001; 18(3):169-75.

38. Asakura H, Hashimoto T, Zenda S, et al. Analysis of dose-volume histogram parameters for radiation pneumonitis after definitive concurrent chemoradiotherapy for esophageal cancer. Radiother Oncol. 2010; 95(2):240-4.

39. Kwa SL, Lebesque JV, Theuws JC, et al. Radiation pneumonitis as a function of mean lung dose: an analysis of pooled data of 540 patients. Int J Radiat Oncol Biol Phys. 1998; 42(1):1-9.

40. Wang S, Liao Z, Wei X, et al. Association between systemic chemotherapy before chemoradiation and increased risk of treatment-related pneumonitis in esophageal cancer patients treated with definitive chemoradiotherapy. J Thorac Oncol. 2008; 3(3):277-82.

41. Reynolds JV, Ravi N, Hollywood D, et al. Neoadjuvant chemoradiation may increase the risk of respiratory complications and sepsis after transthoracic esophagectomy. $J$ Thorac Cardiovasc Surg. 2006; 132(3):549-55.

42. Elliott JA, Murphy CF, Doyle SL, et al. The effect of neoadjuvant chemoradiation on preoperative pulmonary physiology, postoperative respiratory complications and quality of life in patients with oesophageal cancer. Br J Surg. 2019; in press.

43. Hoeppner J, Lordick F, Brunner T, et al. ESOPEC: prospective randomized controlled multicenter phase III trial comparing perioperative chemotherapy (FLOT protocol) to neoadjuvant chemoradiation (CROSS protocol) in patients with adenocarcinoma of the esophagus (NCT02509286). BMC Cancer. 2016; 16:503.

44. Reynolds JV, Preston SR, O'Neill B, et al. ICORG 10-14: NEOadjuvant trial in Adenocarcinoma of the oEsophagus and oesophagoGastric junction International Study (Neo-AEGIS). BMC Cancer. 2017; 17(1):401.

45. Low DE, Alderson D, Cecconello I, et al. International consensus on standardization of data collection for complications associated with esophagectomy: Esophagectomy Complications Consensus Group (ECCG). Ann Surg. 2015; 262(2):286-94.

46. Goense L, Meziani J, Bulbul M, et al. Pulmonary diffusion capacity predicts major complications after esophagectomy for patients with esophageal cancer. Dis Esophagus. 2019. https://d oi.org/10.1093/dote/doy082.

Publisher's Note Springer Nature remains neutral with regard to jurisdictional claims in published maps and institutional affiliations. 